\title{
Chaos Synchronization of a Class of Chaotic Systems via Linear State Error Feedback Control
}

\author{
Liming $\mathrm{DU}^{\mathrm{a}}{ }^{*}$, Fengying $W A N \mathrm{G}^{\mathrm{b}}$, Ziyang $\mathrm{HAN}^{\mathrm{c}}$ and Jie DONG ${ }^{\mathrm{d}}$ \\ Faculty of Information \& Control Engineering, Shenyang Jianzhu University, Shenyang Liaoning, \\ 110168, China. \\ aduliboy@163.com, ’wangfy678@163.com, 'phenixhans@163.com, `xxdongjie@sjzu.edu.cn, \\ ${ }^{*}$ corresponding author
}

Keywords: synchronization; chaos; linear feedback

\begin{abstract}
This paper studies the synchronization problem of a class of chaotic systems. The mentioned synchronization scheme consists of identical master and slave chaotic systems. By analyzing structural characteristics of nonlinear vector field of the error system, a new approach is presented to synchronize the two chaotic systems. Based on linear error state feedback, an independent stable equation of some error state variables is separated. Synchronization conditions is derived which make globally synchronization between the master system and the slave system rapidly. The approach can be used to achieve synchronization of the Genesio chaotic system and Liu chaotic system. A class of simple linear feedback controller is designed and precise corresponding synchronization conditions is obtained. Numerical examples illustrate the validity of the presented approach in this paper.
\end{abstract}

\section{Introduction}

During the past decades, chaos plays a more and more important role in nonlinear science field. A chaotic system has complex dynamical behaviors, extreme sensitivity to tiny variations of initial conditions is a prominent characteristic of chaotic behavior [1-2]. As a topic of dynamical systems theory, chaos synchronization has received a significant attention, since Pecora and Carroll presented the chaos synchronization method to synchronize two identical chaotic systems with different initial values in 1990[3]. A basic configuration for chaos synchronization is the master-slave pattern, where the slave chaotic system must track the master chaotic trajectory. A number of methods based on this configuration have been proposed[4-14], such as feedback approach [4,5,6], time-delay feedback approach $[7,8,9]$, adaptive method [10,11], backstepping design technique [12,13], Impulse control method [14], sliding mode control method[15] and so on.

On the other hand, due to these nonlinear chaotic systems including many quadratic nonlinear terms, most of the synchronization controller is nonlinear. So, it is not conducive to engineering applications. Considering linear state error feedback control is a robust and easily implemented control technique available for chaos synchronization. Thus, in recent years, this technique has been used to synchronize two identical classical Lorenz systems[16,17] and Chen systems[18]. Some sufficient synchronization conditions have been derived. However, most of the results only for very specific chaotic system, and most of the linear controllers providing rather conservative.

This paper systematically investigates chaos synchronization of a class of chaotic systems via linear state error feedback control. By analyzing the nonlinear vector fields of the error system between drive and response system, all of components are associated with a component of error state. Hence, when this state component tends to zero, the nonlinear part is gradually disappearing. If the component of the error state is controlled to zero, the nonlinear error system is becoming controlled linear system, and if this linear system is globally asymptotically stable at the zero equilibrium point, chaos synchronization is achieved. It should be noted that the method is simple, fast and effective, and it can be applied to get synchronization of Genesio chaotic system and Liu chaotic systems, some simple linear feedback controller is designed and precise corresponding synchronization conditions is 
obtained. Numerical examples illustrate the validity of the presented approach in this paper.

\section{The synchronization scheme}

Consider a class of chaotic systems described by

$$
\dot{x}=A x+f(x),
$$

Where $x=\left[x_{1}, x_{2}, \ldots, x_{n}\right]^{T}$ is the state vector of the chaotic system, $A=R^{n \times n}$ is a constant matrix, $f(x)=\left[f_{1}(x), f_{2}(x), \cdots, f_{n}(x)\right]^{T}$ is the nonlinear vector fields with $f(0)=0$. As we known, many chaotic systems investigated are in the form (1) such as the unified chaotic system, Lorenz system, Chen system, Chua's circuit, and so on.

Now, construct a master-slave synchronization scheme for two identical chaotic systems coupled by a linear state error feedback controller $u(t)$, as follows:

$$
\left\{\begin{array}{l}
\text { Master }: \dot{x}=A x+f(x) \\
\text { Slave }: \dot{y}=A y+f(y)+u(t)
\end{array}\right.
$$

Then the objective of the synchronization scheme (2) is to design a controller such that the trajectories $x$ of the master and the trajectories $y$ of the slave systems satisfy.

$$
\lim _{t \rightarrow \infty}\|y(t)-x(t)\|=0 \text {. }
$$

Define the error variable $e=y-x$, where $e=\left(e_{1}, e_{2}, e_{3}\right)^{T}=\left(y_{1}-x_{1}, y_{2}-x_{1}, y_{3}-x_{3}\right)^{T}$, from (2), we can abtain a dynamical error system:

$$
\dot{e}=A e+f(y)-f(x)+u(t)
$$

In order to find a sufficient synchronization criterion for the scheme, the following assumption on the error system is needed.

Assumption 1. Consider the case that there exist a constant $i$, and the nonlinear parts of (4) have the following condition:

(1) $f_{i}(y)-f_{i}(x)=0$,

(2) $f(y)-f(x)=e_{i} g(x, y)+M(x) e$,

Where $M(x)=\left[m_{i j}(x)\right] \in R^{n \times n}$, and $m_{i}(x)=0, g(x, y)=\left[g_{1}(x, y), g_{2}(x, y), \cdots g_{n}(x, y)\right]^{T}$ is the nonlinear vector fields with $g_{i}(x, y)=0$.

It is easy to verify that many chaotic systems satisfy the conditions of assumption 1 , such as Genesio system, Lorzen chaotic system, Chen system and Liu chaotic system.

Let designs the controller $u(t)$ as the following:

$$
u(t)=K e,
$$

Where $K \in R^{n \times n}$ is a constant matrix, referred to as the feedback gain matrix. Hence, system (4) can be expressed as

$$
\dot{e}=(\hat{A}+M(x)) e+e_{i} g(x, y),
$$

Where $\hat{A}=A+K=\left[\hat{a}_{i j}\right] \in R^{n \times n}$. From the viewpoint of control theory, the synchronization issue (3) is equivalent to the uniform asymptotical stability of the error system (6) at $e=0$.

\section{Main theoretical results}

In this section, we will develop some globally synchronization criteria by the feedback control law.

Theorem 1. The master-slave synchronization scheme (2) achieves synchronization under the assumption 1, if there exist a constant matrix $K \in R^{n \times n}$ to make the following two condition satisfied:

(1) The matrix $\bar{A}(x)=\hat{A}+M(x)$ is Hurwitz stability for all orbits $x$ of the master system.

(2) $\dot{e}_{i}=\hat{a}_{i i} e_{i}$.

Proof. For an independent $\dot{e}_{i}=\hat{a}_{i i} e_{i}, \quad f_{i}(y)-f_{i}(x)=0$, system (6) can be rewritten as follow: 


$$
\dot{e}=\left[\begin{array}{ccccc}
\hat{a}_{11} & \cdots & \cdots & \cdots & \hat{a}_{1 n} \\
\cdots & \ddots & \cdots & \cdots & \cdots \\
0 & \cdots & \hat{a}_{i i} & \cdots & 0 \\
\cdots & \cdots & \cdots & \ddots & \cdots \\
\hat{a}_{n 1} & \cdots & \cdots & \cdots & \hat{a}_{n n}
\end{array}\right] \times\left[\begin{array}{c}
e_{1} \\
\vdots \\
e_{i} \\
\vdots \\
e_{n}
\end{array}\right]+\left[\begin{array}{ccccc}
m_{11}(\mathrm{x}) & \cdots & \cdots & \cdots & \mathrm{m}_{1 n}(x) \\
\cdots & \ddots & \cdots & \cdots & \cdots \\
0 & \cdots & 0 & \cdots & 0 \\
\cdots & \cdots & \cdots & \ddots & \cdots \\
m_{n 1}(\mathrm{x}) & \cdots & \cdots & \cdots & m_{n n}(x)
\end{array}\right] \times\left[\begin{array}{c}
e_{1} \\
\vdots \\
e_{i} \\
\vdots \\
e_{n}
\end{array}\right]+e_{i}\left[\begin{array}{c}
g_{1}(x, y) \\
\vdots \\
0 \\
\vdots \\
g_{n}(x, y)
\end{array}\right]
$$

For $\bar{A}(x)$ is Hurwitz stability for all orbits $x$ of the master system, so $\operatorname{Re} \lambda(\bar{A}(x))<0$, where $\lambda(\bar{A}(x))=\left\{\lambda_{1}(x), \lambda_{2}(x) \cdots, \lambda_{n}(x)\right\}$ is eigenvalue of $\bar{A}(x)$. Obviously, $\lambda_{i}(x)=\hat{a}_{i i}<0, \dot{e}_{i}=\hat{a}_{i i} e_{i}$. Hence, when $t \rightarrow \infty, e_{i} \rightarrow 0$ and $e_{i} g(x, y) \rightarrow 0$. So the system (7) degenerate gradually into stabile linear system $\dot{e}=\bar{A}(x) e . \bar{A}(x)$ is Hurwitz stability, system $\dot{e}=\bar{A}(x) e$ is globally asymptotical stabile at $e=0$, so the master-slave synchronization scheme (2) achieves synchronization, which complete the proof.

Remark 1. If $M(x)=0$, then the first condition in theorem 1 will be simplified as $\hat{A}=A+K$ is Hurwitz stability.

Remark 2. For all the state variable $x$ of chaotic system is bounded, so it is easy to verfy the condition(1) in Theorem 1.

Although, the criterion of Theorem 1 is very simple, and we need not construct any Lyapunov function or solving any matrix inequalities, but to get appropriate feedback gain matrix $K$, we need to correct estimate the boundary of the chaotic system. To make it easier to design the controller of (7), we consider the case that the matrix $M(x)$ has the following special structure.

$$
M(x)=\left[\begin{array}{ccccc}
0 & \cdots & \cdots & 0 & m_{1 n}(x) \\
\vdots & \ddots & & \vdots & \vdots \\
\vdots & & \ddots & \vdots & \vdots \\
0 & \cdots & \cdots & 0 & m_{(n-1) n} \\
-m_{1 n}(\mathrm{x}) & \cdots & - & -m_{(n-1) n}(\mathrm{x}) & 0
\end{array}\right] .
$$

It is easy to find many chaotic systems satisfy the condition (8).

\section{Main application}

Synchronization of Genesio system. Genesio system is a very useful chaotic system, the master-slave synchronization scheme for Genesio system can be write as form (2), where $A=\left[\begin{array}{ccr}0 & 1 & 0 \\ 0 & 0 & 1 \\ -\alpha & -\beta & -\gamma\end{array}\right], \alpha>0, \beta>0, \gamma>0, f(x)=\left[\begin{array}{l}0 \\ 0 \\ x_{1}^{2}\end{array}\right], \quad f(y)=\left[\begin{array}{l}0 \\ 0 \\ y_{1}^{2}\end{array}\right]$, and $u(t)$ is the controller as the form of

(5). Then the error dynamic system can be derived into a form as

$$
\dot{e}=A e+e_{1}\left[\begin{array}{c}
0 \\
0 \\
y_{1}+x_{1}
\end{array}\right]+u(t) \text {. }
$$

It is easy to see that system (12) satisfies Assumption 1. Now, design the controller as.

$$
u=K e \text {, }
$$

where $K=\left[\begin{array}{ccc}k_{11} & -1 & 0 \\ 0 & \mathrm{k}_{22} & -1 \\ 0 & 0 & 0\end{array}\right]$.

Theorem 2. If $k_{11}<0, k_{22}<0$, the error dynamic system(9) is global asymptotical stable with the controller (10), that is to say, the master-slave Genesio system (2) achieves global synchronization via the linear state error feedback controller.

Proof. With the controller (10), we have 


$$
\begin{aligned}
& \bar{A}(x)=A+K=\hat{A} \\
& =\left[\begin{array}{lcc}
0 & 1 & 0 \\
0 & 0 & 1 \\
-\alpha & -\beta & -\gamma
\end{array}\right]+\left[\begin{array}{ccc}
k_{11} & -1 & 0 \\
0 & \mathrm{k}_{22} & -1 \\
0 & 0 & 0
\end{array}\right]=\left[\begin{array}{lcc}
k_{11} & 0 & 0 \\
0 & k_{22} & 0 \\
-\alpha & -\beta & -\gamma
\end{array}\right] .
\end{aligned}
$$

The eigenvalues of $\hat{A}$ are

$$
\begin{aligned}
& \lambda_{1}=k_{11}<0, \\
& \lambda_{2}=k_{22}<0, \\
& \lambda_{3}=-\gamma<0 .
\end{aligned}
$$

Hence, the matrix $\bar{A}$ is is Hurwitz stability and $\dot{e}_{1}=\hat{a}_{11} e_{1}$, According to the Theorem 1, the Genesio system achieves synchronization, which complete the proof.

Synchronization of Liu chaotic systems. In this section, we study the synchronization of Liu chaotic systems by linear error state feedback controller. Now, write the master-slave Liu system as form (2), we get $A=\left[\begin{array}{ccc}-a & 0 & a \\ 0 & -b & 0 \\ c & 0 & 0\end{array}\right]$, where $a>0, b>0, c>0, f(x)=\left[\begin{array}{c}0 \\ h x_{1}^{2} \\ -d x_{1} x_{2}\end{array}\right], f(y)=\left[\begin{array}{c}0 \\ h y_{1}^{2} \\ -d y_{1} y_{2}\end{array}\right]$. Then the error dynamic system can be described as

$$
\dot{e}=A e+M(x) e+e_{1}\left[\begin{array}{c}
0 \\
0 \\
y_{1}+x_{1}
\end{array}\right]+u(t)
$$

Where $M(x)=\left[\begin{array}{ccc}0 & 0 & 0 \\ 0 & 0 & 0 \\ 0 & x_{1} & 0\end{array}\right]$. Let design the controller as

$$
u=K e,
$$

where $K=\left[\begin{array}{ccc}0 & 0 & -a \\ 0 & 0 & 0 \\ 0 & 0 & k_{33}\end{array}\right]$.

Theorem 3. If $k_{33}<0$, the master-slave Liu system (2) achieves synchronization via the linear state error feedback controller (13).

Proof. Applying the controller (13), we obtain

$\bar{A}(x)=A+K+M(x)$

$=\left[\begin{array}{ccc}-a & 0 & a \\ 0 & -b & 0 \\ c & 0 & 0\end{array}\right]+\left[\begin{array}{lll}0 & 0 & -a \\ 0 & 0 & 0 \\ 0 & 0 & k_{33}\end{array}\right]+\left[\begin{array}{lll}0 & 0 & 0 \\ 0 & 0 & 0 \\ 0 & x_{1} & 0\end{array}\right]=\left[\begin{array}{ccc}-a & 0 & 0 \\ 0 & -b & 0 \\ c & x_{1} & k_{33}\end{array}\right]$.

Obviously, the eigenvalues of $\bar{A}(x)$ are

$$
\begin{aligned}
& \lambda_{1}=-a<0, \\
& \lambda_{2}=-b<0, \\
& \lambda_{3}=c+k_{33}<0 .
\end{aligned}
$$

So the matrix $\bar{A}(x)$ is is Hurwitz stability and $\dot{e}_{1}=\hat{a}_{11} e_{1}$, According to the Theorem 1, the Liu system achieves synchronization.

\section{Numerical simulation}

Genesio system. According to Theorem 2, if we take $\alpha=6, \beta=2.92, \gamma=1.2, k_{11}=-1, k_{22}=-2$, the synchronization of Genesio system is achieved. The state trajectories of master and slave Genesio systems are showed as Fig.1. 


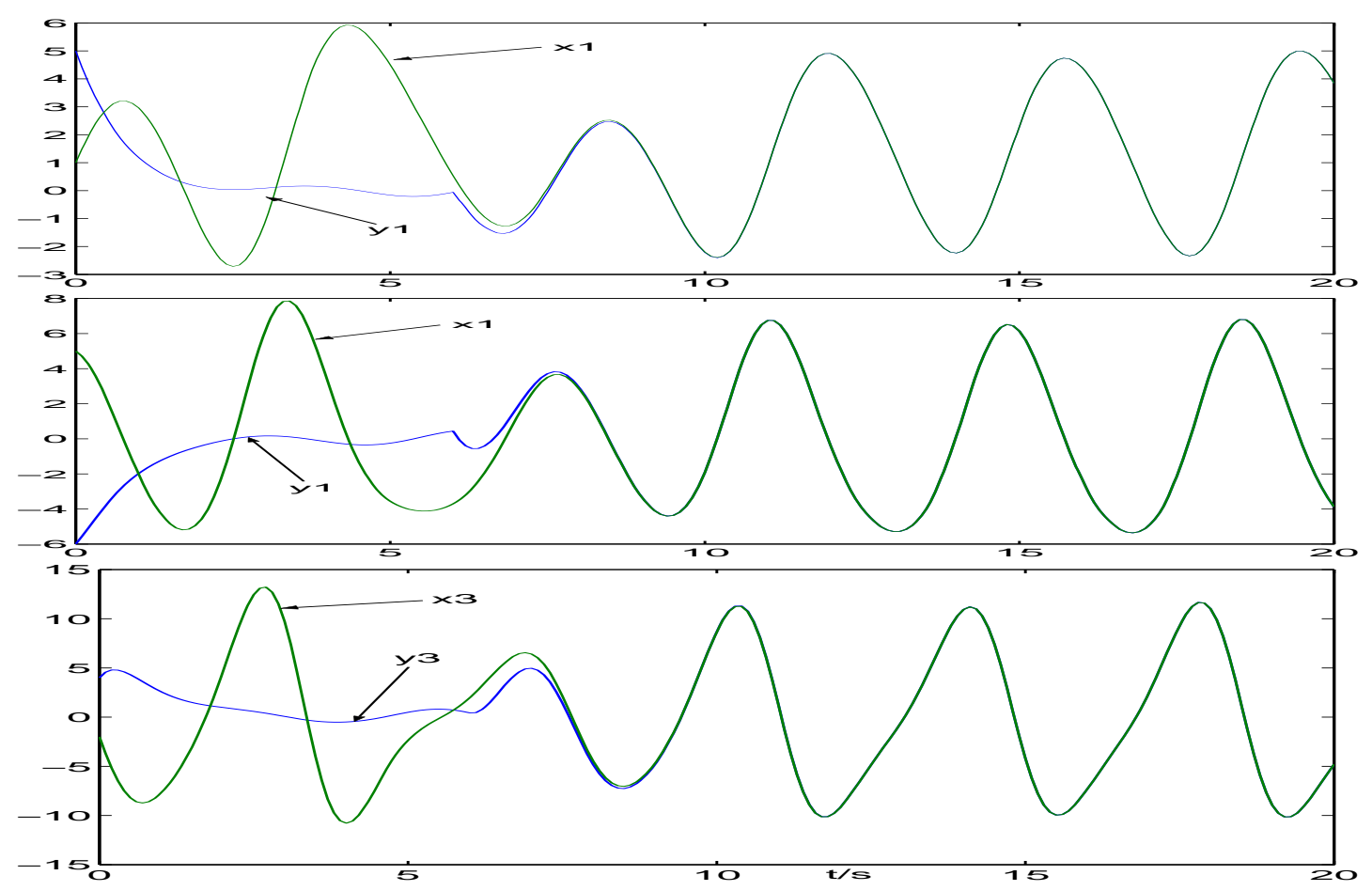

Fig.1. state trajectories of master and slave Genesio systems

Form fig.1, it is easy to see that after the controller is used in 6 second, the trajectory of slave system tracking the trajectory of master system quickly, which demonstrates the validity of Theorem 3.

Liu chaotic systems. To achieved synchronization of Liu systems, we select $a=4, b=2, \beta=28, k_{33}=-2$. It is easy to verfy that conditions of theorem 3 are satisfied. The state trajectories of master and slave Liu systems are showed as Fig.2.

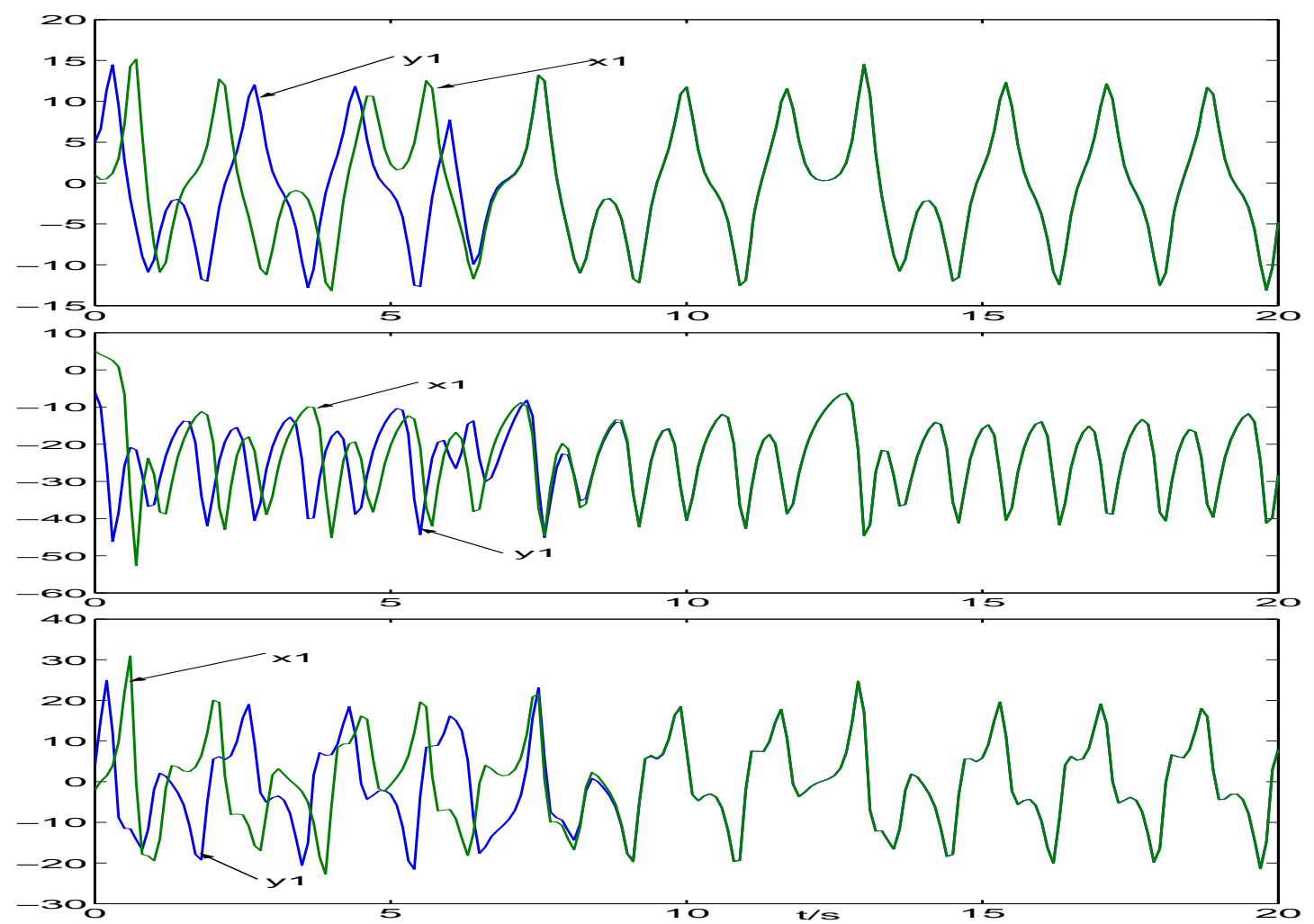

Fig.2. state trajectories of master and slave Liu systems 


\section{Conclusion}

The paper provides a promising new approach for synchronization of chaotic systems via the simple but effective linear state error feedback control. Some new sufficient synchronization criteria have been obtained. This approach has used to achieve synchronization of the Genesio chaotic system, Lorzen chaotic system, Chen system and Liu chaotic system. Numerical examples illustrate the validity of the preseneted method in this paper.

\section{Acknowledgement}

This work was supported by the National Natural Science Foundation of China under Grants 61272253 and Shenyang Jianzhu University Subject Culvert Project XKHY2-72.

\section{References}

[1] Fradkov AL , Evans RJ. Control of chaos: Methods and applications in engineering[J]. Annual Reviews in Control. 2005:29(1):33-56.

[2] Ott E , Grebogi C, Yorke JA.Controlling chaos[J]. Phys Rev Lett. 1990:64(11):1196-1199.

[3] Pecora LM, Carroll TL. Synchronization in chaotic systems[J]. Phys Rev Lett, 1990: 64(8): $821-824$.

[4] Matouk AE. Chaos feedback control and synchronization of a fractional-order modified Autonomous Van der Pol-Duffing circuit[J]. Communications in Nonlinear Science and Numerical Simulation. 2011;16(2):975-986.

[5] Lu J, Wu X, Han X, Lü J. Adaptive feedback synchronization of a unified chaotic system[J]. Phys Lett A. 2004:329:327-333.

[6] Fu SH, Pei LJ. Chaotic synchronization of Chua s circuits with nonlinear control[J]. Acta Phys. Sin. 2010: 59(9):5985`5989.

[7] Park JH, Kwon OM. A novel criterion for delayed feedback control of time-delay chaotic systems. Chaos[J]. Solitons \& Fractals. 2005;23(2):495-501.

[8] Ghosh D, Banerjee S, Chowdhury AR, Generalized and projective synchronization in modulated time-delayed systems[J]. Physics Letters A, 374(21):2143-2149, 2010.

[9] Li C, Liao XF, Kwokwo W. Chaotic lag synchronization of coupled time-delayed systems and its applications in secure communication[J]. Physica D,2004:194(3):187-202.

[10] Park JH. Adaptive synchronization of a unified chaotic systems with an uncertain parameter[J]. Int J Nonlin Sci Numer Simulat. 2005:6:201-206.

[11] Hu M, Xu Z,Adaptive feedback controller for projective synchronization[J]. Nonlinear Analysis: Real World Applications. 2008:9(3):1253-1260.

[12] Wu XQ, Lu JA. Parameter identification and backstepping control of uncertain Lu system. Chaos[J]. Solitons \& Fractals. 2003;18(4):721-729.

[13] Li YS, Lu L, Liu Y. Spatiotemporal chaos synchronization of complex networks by backstepping design[J]. Acta Phys. Sin. 2013: 62(2).

[14] Khadra A, Liu X, Shen X. Application of impulsive synchronization to communication security[J]. IEEE Trans. On Circuits and Systems. 2003:50(3):341-350.

[15] Yau HT. Chaos synchronization of two uncertain chaotic nonlinear gyros using fuzzy sliding mode control[J]. Mechanical Systems and Signal Processing. 2008:22(2): 408-418. 
[16] Lü J, Zhou T, Zhang S. Chaos synchronization between linearly coupled chaotic systems[J]. Chaos Solitons Fractals. 2002:14:29-541.

[17] Li D, Lu JA., Wu XQ. Linearly coupled synchronization of the unified chaotic systems and the Lorenz systems[J]. Chaos Solitons Fractals. 2005:23: 79-85.

[18] Wang Y, Guan ZH, Wang HO. Feedback and adaptive control for the synchronization of Chen system via a single variable[J]. Phys. Lett. A. 2003:312: 34-40. 